\title{
Outbreak of cryptosporidiosis in a child day-care centre in Gipuzkoa, Spain, October to December 2011
}

J Artieda (epidem2-san@ej-gv.es) ${ }^{1}$, M Basterrechea ${ }^{1}$, L Arriola ${ }^{1}$, M Yagü $^{1,2}$, E Albisua ${ }^{1}$, N Arostegui $^{3}$, U Astigarraga ${ }^{3}$, R Botello $^{3}$, J M Manterola 4

1. Public Health Division of the Basque Government, Gipuzkoa, Spain

2. Unit of Preventive Medicine and Public Health, Cruces Hospital, Barakaldo, Spain

3. Health Centre of Osakidetza (Basque Health Service), Eibar, Gipuzkoa, Spain

4. Microbiology Department, Mendaro Hospital, Mendaro, Spain

Citation style for this article:

Artieda J, Basterrechea M, Arriola L, Yagüe M, Albisua E, Arostegui N, Astigarraga U, Botello R, Manterola JM. Outbreak of cryptosporidiosis in a child day-care

centre in Gipuzkoa, Spain, October to December 2011.

Euro Surveill. 2012;17(5):pii=20070. Available online: http://www.eurosurveillance.org/ViewArticle.aspx?Articleld=20070

Article published on 2 February 2012

From October to December 2011, an outbreak of 26 cases of cryptosporidiosis occurred in a day-care centre in Gipuzkoa, Spain. The infection spread from person to person and affected 24 children under two years of age (attack rate: $38 \%$ ) and two caregivers. Cryptosporidium oocysts were observed in 10 of 15 samples. During 2010, only four cases of cryptosporidium were detected in Gipuzkoa, and 27 overall in Spain.

On 24 November 2011, a paediatrician notified the epidemiological surveillance service of Gipuzkoa (Basque Country, northern Spain) of a child with diarrhoea in whose stools oocysts of Cryptosporidium had been isolated, as well as of an unusually large number of children with diarrhoea who attended the same day-care centre as the first child. All were tested for Cryptosporidium because our laboratory has a policy of testing for this microorganism in samples from children under the age of five years. In this paper, we present the epidemiological, environmental and parasitological research undertaken to study the outbreak and report the measures taken to control it.

\section{Background}

Cryptosporidium is a coccidian parasite. Its infectious forms, oocysts, are excreted in the host's faeces. The principal zoonotic reservoirs are humans, cattle and other domestic animals. It is transmitted by the faecal-oral route: person-to-person or from animal to person, as well as by ingestion of contaminated water or food. Extensive outbreaks have been reported to be associated with transmission through drinking water or related to swimming pools $[1,2]$. On the other hand, transmission between humans has resulted in outbreaks in day-care centres with incidence rates of $30-60 \%$ [3-7]. Given that oocysts are resistant to chlorine, it is essential that properly functioning filtration systems are used for the safety of public water supplies [8].
The median incubation period in humans is seven days (range: 2-14 days). Oocysts are found in stools an average of seven days after the end of signs and symptoms, and in most cases they stop being excreted two weeks after symptoms have resolved. The most common signs and symptoms include watery diarrhoea, abdominal pain, vomiting and fever. In immunocompetent patients, the infection is self-limiting, lasting for up to 20 days (mean of 10 days) [7].

During 2010 and the first 25 weeks of 2011, 46 cases of cryptosporidiosis were notified to the Spanish National Microbiology Surveillance System. Thirty-one of these cases were children aged between one and four years, followed by nine children aged five to nine years [9]. Data from other countries in Europe are diverse and notification rates during 2009 vary considerably between countries, with 10 per 100,000 in Ireland, 4.37 per 100,000 in Belgium and 1.35 per 100,000 in Germany [10].

\section{Outbreak investigation}

An active search for cases in the day-care centre was undertaken, by three primary care paediatricians and the Microbiology Unit of the referral hospital. A case was defined as a child or staff of the day-care centre who presented between 1 October and 20 December 2011 with frequent, non-bloody, watery diarrhoea, and/or in whose stool Cryptosporidium oocysts had been isolated. The following variables were recorded for the detected cases: sex, age, date of onset, clinical signs and symptoms, diarrhoea in people living in the same household, and complications.

Samples were taken for microbiological and parasitological analysis. Cryptosporidium oocysts were detected by extension on microscope slides, drying, Auramine 0 staining and observation at $400 x$ magnification in an epifluorescence microscope. The samples were also investigated for the following 
microorganisms: Salmonella, Shigella, Campylobacter, Aeromonas and Yersinia enterocoliticus. The laboratory does not look systematically for viruses until the number of suspected cases increases in the population. Cases went up in our community in the last week of December 2011.

The epidemic curve confirmed that an outbreak was ongoing and showed a person-to-person pattern of transmission (Figure). Twenty-six individuals fulfilled the case definition, with onset of symptoms on 14 October in the first case and on 6 December in the last. All those affected presented with diarrhoea and the duration of illness was five to 30 days, with irregular occurrence of symptoms. All except two of the children were seen by a paediatrician and none received drug treatment. The day-care centre occupies a three-story building, with two classrooms on each floor.

At the time of the study, 63 children between o and two years of age attended the day-care, as well as the staff that consisted of six caregivers. There were 39 $1-2$-year-olds in classroom 2 (ground floor) and classrooms 3 and 4 (first floor), 13 in each. In classroom 1 (ground floor) and classrooms 5 and 6 (second floor), there were $240-1$-year-olds, eight in each. A total of 24 children fell ill (attack rate: $38.1 \%$ ), and only three of them were in the group of $0-1$-year-olds. Children shared some activities by age group. The Table lists the number of children affected and the attack rates in each classroom. Two caregivers also fell ill.

Three household contacts reported diarrhoea during the outbreak period, but their aetiology was not determined. There were no complications except in a pair of two siblings who both lost weight. In the microscopic analysis, Cryptosporidium spp. oocysts were isolated in 10 of 15 stool samples, and no other enteropathogen was found in any of the samples studied.

\section{FIGURE}

Cases of diarrhoea caused by Cryptosporidium spp. in a day-care centre, Gipuzkoa, Spain, October-December 2011 $(\mathrm{n}=26)$

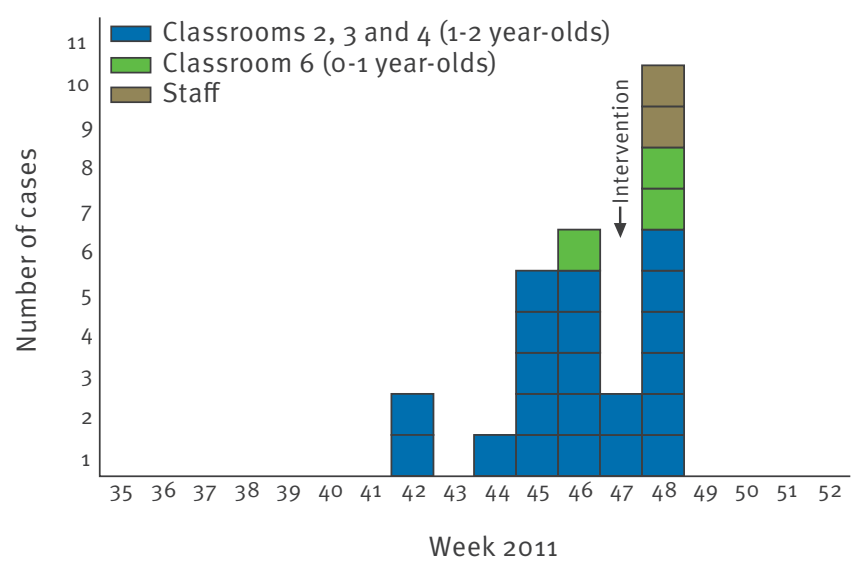

\section{Environmental investigation and control measures}

In addition, an environmental investigation was also undertaken by the local public health technicians. Information on hygiene practices and water usage was collected. The investigation detected deficiencies in hygiene procedures in the day-care centre. Single use paper towels were not available in any of the risk areas. Besides, the hot water system was damaged during the period of the outbreak and only cold water was available.

As soon as the outbreak was confirmed, strengthening of hygiene measures was recommended to the staff of the day-care centre, and they were asked to advise taking children to their paediatrician in the event of more cases. The recommended measures involved correcting the above-mentioned deficiencies, improving compliance with universal hygiene rules and, given the characteristics of the microorganism (resistance to chlorine), cleaning surfaces with $3 \%$ hydrogen peroxide [8]. All measures recommended were implemented within $24-48$ hours.

A letter was sent to the parents informing them of the outbreak and advising good hygiene practices. In addition, they were told that those with diarrhoea must not to use public swimming pools or other recreational water facilities for the duration of the outbreak $[7,8]$.

\section{Discussion}

Although it is assumed that the most common transmission is through water [11], water was not considered in the outbreak described here because no additional cases were detected in the local population, the children did not engage in water activities and they only drank bottled water. The epidemiological curve shows that the first two cases occurred in October 2011 in the same classroom (ages 1-2 years), and that it was transmitted to other children that shared the same classroom and/or activities. In the classrooms of the 0-1-year-olds, only three cases occurred; it is important to note that the index case was a relative of a case

\section{TABLE}

Number of cryptosporidiosis cases and attack rate in each classroom of a day-care centre, Gipuzkoa, Spain, OctoberDecember $2011(n=24)$

\begin{tabular}{|l|c|c|c|c|}
\hline Classroom & $\begin{array}{c}\text { Age } \\
\text { (years) }\end{array}$ & $\begin{array}{c}\text { Number of } \\
\text { exposed }\end{array}$ & $\begin{array}{c}\text { Number of } \\
\text { cases }\end{array}$ & Attack rate \\
\hline $\begin{array}{l}\text { Ground floor } \\
\text { Classroom 1 }\end{array}$ & $0-1$ & 8 & 0 & $0 \%$ \\
Classroom 2 & $1-2$ & 13 & 4 & $31 \%$ \\
\hline First floor & & & & \\
Classroom 3 & $1-2$ & 13 & 10 & $77 \%$ \\
Classroom 4 & $1-2$ & 13 & 7 & $54 \%$ \\
\hline Second floor & & & & $0 \%$ \\
Classroom 5 & $0-1$ & 8 & 0 & $37.5 \%$ \\
Classroom 6 & $0-1$ & 8 & 3 & $38.1 \%$ \\
\hline Total & & 63 & 24 & \\
\hline
\end{tabular}


in the classrooms of the 1-2-year-olds. Consistent with descriptions in the literature $[7,12]$, the illness in this Cryptosporidium outbreak was mild and self-limiting with a relatively long duration.

It is known that the infective dose for Cryptosporidium is relatively low (one to 10 oocysts) and affected individuals excrete a large number of oocysts (up to $\left.10^{8}\right)^{\star}$ [12]. In our reference hospital, which covers a population of 75,000 inhabitants, four positive Cryptosporidium cases were detected during 2010, and 17 in 2011. Of these 17 cases, 10 were children in the studied daycare centre.

Further, though routine laboratory tests to determine whether stool samples contain parasites and/or eggs do not identify species of Cryptosporidium. On this occasion, the fact that our laboratory has a policy of testing for this microorganism in under-fives made it possible to identify the aetiology of the outbreak. Although national coverage is not guaranteed, 27 cases were notified to the surveillance system in Spain during 2010. Our laboratory, which covers $0.15 \%$ of the Spanish population, notified four cases. This strongly suggests that cryptosporidiosis is an underdiagnosed disease in Spain.

Once the outbreak was declared, efforts were made to detect and remedy problems, as well as the application of stringent hygiene by caregivers as described above, and seemed to be effective in stopping the spread of the infection. Depletion of susceptible hosts could also be considered as a possible reason that stopped the outbreak. Nevertheless, probably thanks to the implemented measures, children under the age of one year were practically not affected, except for those who had close contact with one of the older cases. The Food and Drug Administration (FDA) approved the usage of nitazoxanide as first choice drug against Cryptosporidiosis [8]. However, in this case the drug was not prescribed as it was not readily available and all cases recovered naturally. The possibility of excluding affected children from the centre was considered, but discarded due to the lack of consensus in the literature on its effectivity, as well as the high social cost [3,13-15].

${ }^{\star}$ Erratum: The number 108 was corrected on 3 February 2012.

\section{References}

1. MacKenzie WR, Hoxie NJ, Proctor ME, Gradus MS, Blair KA, Peterson DE, et al. A massive outbreak in Milwaukee of Cryptosporidium infection transmitted through the public water supply, N Engl J Med. 1994;331(3):161-7.

2. Yoder JS, Harral C, Beach MJ; Centers for Disease Control and Prevention (CDC). Cryptosporidiosis Surveillance - United States, 2006-2008; MMWR Surveill Summ. 2010;59(6):1-14.

3. Vandenberg O, Robberecht F, Dauby N, Moens C, Talabani $H$, Dupont E, et al. Management of a Cryptosporidium hominis Outbreak in a Day-care Center. Pediatr Infect Dis J. 2012;31(1):10-15.

4. Heijbel H, Slaine K, Seigel B, Wall P, McNabb SJ, Gibbons W, et al. Outbreak of diarrhea in a day care center with spread to household members: the role of Cryptosporidium. Pediatr Infect Dis J. 1987;6(6):532-5.

5. Tangermann RH, Gordon S, Wiesner P, Kreckman L. An outbreak of cryptosporidiosis in a day-care center in Georgia. Am J Epidemiol. 1991;133(5):471-6.

6. Taylor JP, Perdue JN, Dingley D, Gustafson TL, Patterson M, Reed LA. Cryptosporidiosis outbreak in a day-care center. Am J Dis Child. 1985;139(10):1023-5.

7. American Academy of Pediatrics. Red Book: Enfermedades Infecciosas en Pediatría. Informe del Comité de Enfermedades Infecciosas. [Pediatric Infectious Diseases. Report of the Committee for Infectious Diseases]. 27th ed. Buenos Aires: Médica Panamericana; 2007.

8. Centers for Disease Control and Prevention (CDC). Parasites Cryptosporidium (also known as "Crypto"). Control Measures for Outbreaks. Intensified Cryptosporidiosis (Crypto) Control Measures for the Child Care Setings. Atlanta. CDC. [Accessed 24 Nov 2011]. Available from: http://www.cdc.gov/parasites/ crypto/daycare/outbreak.html

9. Situación de cuatro enfermedades productoras de gastroenteritis a partir de información del Sistema de Información Microbiológica. Primeras 25 semanas de 2011. [Situation of four diseases causing gastroenteritis based on information from the Microbiological Information System. First 25 weeks of 2011]. Informe Semanal de Vigilancia 12 de diciembre de 2011. [Surveillance Weekly Report 12 Dec 2011]. Red Nacional de Vigilancia Epidemiológica. Servicio de Vigilancia Epidemiológica. Centro Nacional de Epidemiología. [National Epidemiological Surveillance Network.

Epidemiological Surveillance Service]. Madrid: Instituto de Salud Carlos III; 2011.

10. European Centre for Disease Prevention and Control. Annual Epidemiological Report 2011. Reporting on 2009 surveillance data and 2010 epidemic intelligence data. Stockholm: ECDC; 2011. Available from: http://ecdc.europa.eu/en/publications/ Publications/1111_SUR_Annual_Epidemiological_Report_on Communicable_Diseases in_Europe.pdf

11. Dillingham RA, Lima AA, Guerrant RL. Cryptosporidiosis: epidemiology and impact. Microbes Infect. 2002;4(10):1059-66.

12. Huang DB, Chappell C, Okhuysen PC. Cryptosporidiosis in children. Semin Pediatr Infect Dis. 2004;15(4):253-9.

13. Brady MT. Infectious disease in pediatric out-of-home child care. Am J Infect Control. 2005;33(5):276-85.

14. Landis SE, Chang A. Child care options for ill children. Pediatrics. 1991;88(4):705-18.

15. Thompson SC. Infectious diarrhoea in children: controlling transmission in the child care setting. J Paediatr Child Health. 1994;30(3):210-9. 